\title{
Ruthenium-106 brachytherapy in treatment of uveal melanoma: 50 years of use!
}

\author{
Sarah Le Hir ${ }^{1}$, and Florence Menetrier ${ }^{1}$ \\ ${ }^{1}$ CEA/DRF/D3P/Prositon, 18 Route du panorama, 92265 Fontenay-aux-Roses Cedex, France
}

Ruthenium-106 $\left({ }^{106} \mathrm{Ru}\right)$ is a beta emitter with a 374 day half-life that is commonly used for brachytherapy of uveal melanoma. Since the beginning of its use in the 1960s, it has been the treatment of choice in Europe unlike North America where it is disfavoured. Compared to other isotopes including beta (Sr-90) and gamma (I-125, Pd-103) emitters, it has a limited penetration depth but the advantage of sparing surrounding normal tissues. Ru-106 plaque radiotherapy has been increasingly used for the treatment of small- to medium-sized tumors up to $5 \mathrm{~mm}$ in thickness [1]. ${ }^{106} \mathrm{Ru}$ plaque provides a maximum activity of $1.6 \mathrm{mCi}$ and a recommended radiation dose to the apex of tumors is $85 \mathrm{~Gy}$ according to a Collaborative Ocular Melanoma Study (COMS) [2].

The local control, non-recurrence and eye-conserving are the main concerns in uveal tumor treatment, besides the patient survival. Though, the radiation threshold dose, effective for the treatment remains unknown and further examinations are needed to design an optimum dose radiation.

Taking into account of geometric tumour parameters, the dose and the time of treatment are directly correlated to the height and basal diameter of tumors, respectively. Software calculations are designed and developed to provide a robust and optimal dose regarding a loss of visual acuity. In fact, dosimetric tolerance levels to macula, optic nerve and retina are evaluated. The Monte Carlo algorithms are used to simulate a dose distribution in the tissues. Indeed, they need to simulate a spectrum resulting from a beta disintegration complicated by a heterogeneous source distribution. Each device used is beforehand calibrated and dose rate in water phantom is given.

Nevertheless, an assessment of dose calculations into plaques points a difference between homogeneous and heterogeneous plaques. A dose distribution of ${ }^{106} \mathrm{Ru}$ plaque and the normalized surface distribution of the emitter substance into a heterogeneous plaque are illustrated on Fig. 1.

A state of art of the use of this radionuclide in terms of dose/response and risk assessment will be presented and the results will be discussed. 


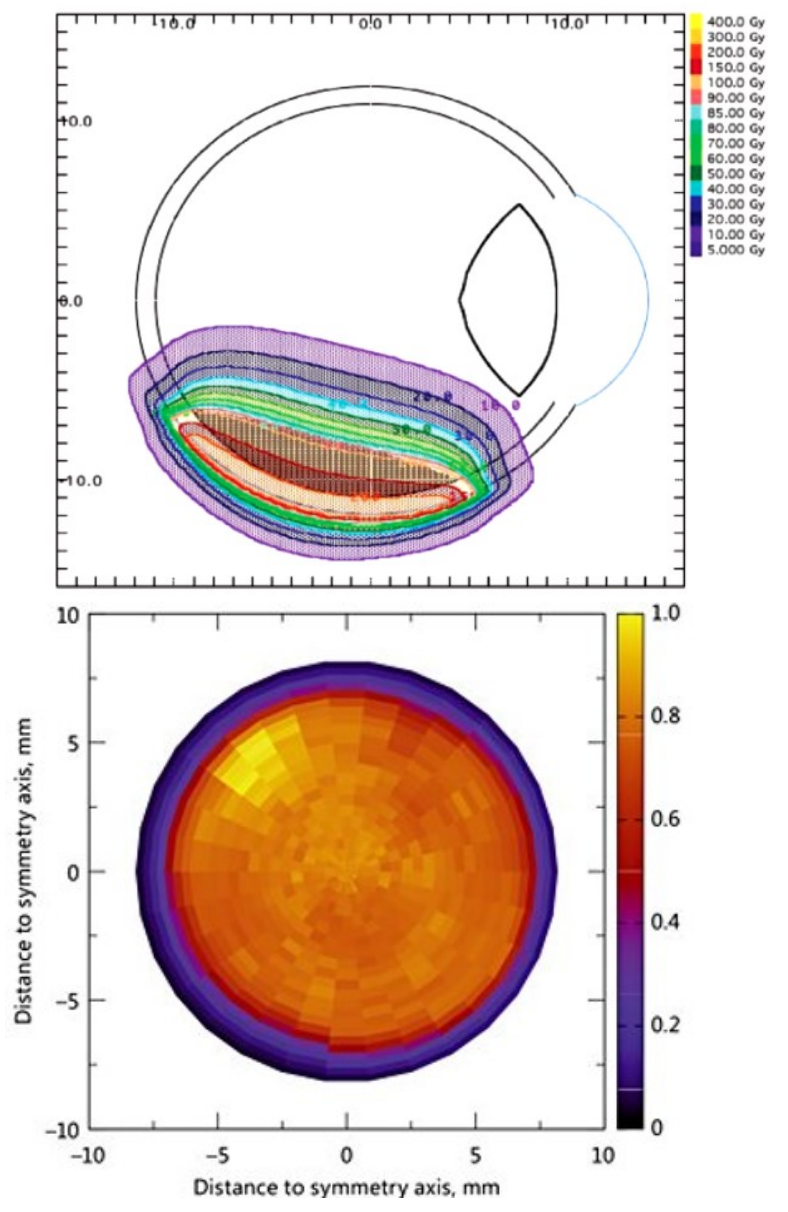

Fig. 1: 2D dose (Gy) distribution for $18 \mathrm{~mm}{ }^{106} \mathrm{Ru}$ plaques [3] (left), experimental emitter dose map for heterogeneous plaque (CCA1364) [4]. 


\section{References}

1. Stannard, C., et al., Radiotherapy for ocular tumours. Eye, 27(2): p. 119127 (2013)

2. Melia, B.M., et al., Collaborative ocular melanoma study (COMS) randomized trial of I-125 brachytherapy for medium choroidal melanoma. I. Visual acuity after 3 years COMS report no. 16. Ophthalmology,. 108(2): p. 348-66 (2001)

3. Wilkinson, D.A., et al., Dosimetric comparison of $R u-106$ and I-125 plaques for treatment of shallow $(<=5 \mathrm{~mm})$ choroidal melanoma lesions. British Journal of Radiology, 81(970): p. 784-789 (2008)

4. Zaragoza, F.J., et al., Monte Carlo Estimation of Absorbed Dose Distributions Obtained from Heterogeneous (106)Ru Eye Plaques. Ocul Oncol Pathol, 3(3): p. 204-209 (2017) 